\title{
Algebraic approach in the study of time-dependent nonlinear integrable systems: Case of the singular oscillator
}

\author{
Jayendra N. Bandyopadhyay*, A. Lakshminarayan and Vijay B. Sheorey \\ Physical Research Laboratory, \\ Navrangpura, Ahmedabad 380 009, India.
}

(October 24, 2018)

\begin{abstract}
The classical and the quantal problem of a particle interacting in onedimension with an external time-dependent quadratic potential and a constant inverse square potential is studied from the Lie-algebraic point of view. The integrability of this system is established by evaluating the exact invariant closely related to the Lewis and Riesenfeld invariant for the time-dependent harmonic oscillator. We study extensively the special and interesting case of a kicked quadratic potential from which we derive a new integrable, nonlinear, area preserving, two-dimensional map which may, for instance, be used in numerical algorithms that integrate the Calogero-Sutherland-Moser Hamiltonian. The dynamics, both classical and quantal, is studied via the timeevolution operator which we evaluate using a recent method of integrating the quantum Liouville-Bloch equations 迎. The results show the exact one-to-one correspondence between the classical and the quantal dynamics. Our analysis also sheds light on the connection between properties of the $S U(1,1)$ algebra and that of simple dynamical systems.
\end{abstract}

PACS numbers : 03.65.-w, 03.65.Fd, 05.45.-a

Typeset using REVTEX

*jayendra@prl.ernet.in 


\section{INTRODUCTION}

The classical and quantum mechanical study of time-dependent Hamiltonian systems are generic and important. They span a wide spectrum of subjects ranging from interaction between atoms and radiation [2, 3], adiabatic [4,5] and non-adiabatic [6.7] Berry phase, timedependent harmonic oscillators [8 10] and quantum motion of a particle in a Paul trap [11,12], to time-dependent mean-field theory (cranking model [13]) in particular and the time-dependent shell model [14] in general.

The question of the existence of invariants (constants of motion) is one of central importance in the study of any dynamical system, be it classical or quantal. A basic theorem of classical mechanics asserts that if the number of independent invariants, satisfying certain conditions, is equal to the number of degrees of freedom, then the motion can be reduced to quadratures, or equivalently, an action-angle transformation to a Hamiltonian dependent only on the actions can be found [15. Such systems are integrable.

The lack of sufficient number of invariants invariably leads to a phase space that has a nonzero measure of chaotic trajectories [16]. For time-independent Hamiltonian systems the Hamiltonian itself is an invariant. However, when the Hamiltonian is an explicit function of time, it is no more an invariant, and this is of course a reflection of the non-conservation of energy. Various methods have been used to obtain approximate solutions for time-dependent problems, e.g., the adiabatic approximation, the sudden approximation, time-dependent perturbation techniques, etc.

The most widely studied time-dependent Hamiltonian system is the time-dependent harmonic oscillator (TDHO). It has long been a problem of considerable interest because of its varied applications in different areas of physics, for instance in molecular physics, quantum chemistry, quantum optics and plasma physics. The Hamiltonian of this system is given by,

$$
\mathcal{H}(t)=\frac{1}{2} p^{2}+\frac{1}{2} \omega^{2}(t) q^{2},
$$

where $p$ and $q$ are conjugate canonical variables. The adiabatic invariant for this system was originally given at the first Solvay Congress in 1911 when the Hamiltonian of this system was used as an approximate Hamiltonian for the slowly lengthening pendulum [17].

The study of this one-dimensional TDHO was greatly advanced due to the work of Lewis [8.9] and Riesenfeld [10]. Lewis [8] determined the exact invariant by applying Kruskal's asymptotic method [18] and showed that a previously known adiabatic invariant was in fact an exact invariant. Later Lewis and Riesenfeld (LR) [10] determined that same invariant by starting with the assumption of the existence of an explicitly time-dependent, homogeneous and quadratic invariant of the form given by,

$$
I(t)=\frac{1}{2}\left[\alpha(t) p^{2}+\beta(t) q^{2}+2 \gamma(t) p q\right],
$$

where the coefficients $\alpha(t), \beta(t)$ and $\gamma(t)$ are time-dependent real functions and $I(t)$ satisfies the condition,

$$
\frac{d I}{d t} \equiv \frac{\partial I}{\partial t}+\{I, \mathcal{H}(t)\}=0 .
$$


Here $\{*, *\}$ denotes the usual Poisson bracket. From the above two equations and after some calculations they derived the exact invariant as,

$$
I(t)=\frac{1}{2}\left[\rho^{2} p^{2}+\left(\dot{\rho}^{2}+\frac{1}{\rho^{2}}\right) q^{2}-2 \rho \dot{\rho} p q\right],
$$

with $\rho(t)$ satisfying the subsidiary condition

$$
\ddot{\rho}+\omega^{2}(t) \rho-\frac{1}{\rho^{3}}=0 .
$$

This more complicated, nonlinear, differential equation represented an advance due to the reason that any particular solution $\rho(t)$ of the above equation would give the exact invariant $I(t)$ for all initial conditions of $p$ and $q$.

The above technique actually predates the work of Lewis and Riesenfeld in a slightly different context, and we briefly point this out. We note that the equation of motion of the TDHO is, $d^{2} q / d t^{2}+\omega^{2}(t) q=0$. This is of the same form as the time-independent, one-dimensional Schrödinger equation, if we assume that $t$ represents the spatial coordinate and $q$, the wave-function. According to an early work by Milne (1930) [19], it is possible to solve this Schrödinger equation using the knowledge of any particular solution of the Eq. (5), after proper identifications. Therefore, any particular solution of the subsidiary condition can also give the exact solution for the TDHO.

Lewis [9] attempted to give an interpretation of $I(t)$ as the most general homogeneous quadratic invariant possible for the Hamiltonian of the one-dimensional TDHO. A more natural and physical interpretation has been suggested by Eliezer and Gray [20] in terms of two-dimensional auxiliary motion, i.e., in terms of a two-dimensional uncoupled TDHO. They showed that the above subsidiary condition Eq. (5) is the radial equation of motion for this two-dimensional system and the invariant $I(t)$ is proportional to the conserved angular momentum of this auxiliary motion. Günther and Leach 21] interpreted $I(t)$ in terms of canonical transformations and under their transformation the invariant $I(t)$ became the Hamiltonian of the one-dimensional time-independent harmonic oscillator of unit frequency.

Besides these previous interpretations of $I(t)$, we can interpret the form of $I(t)$ chosen by Lewis and Riesenfeld (LR) [10] from the Lie-algebraic point of view. The Hamiltonian of the one-dimensional TDHO is formed by the dynamical variables $\frac{1}{2} p^{2}$ and $\frac{1}{2} q^{2}$. These two dynamical variables together with $p q$ are generators of the closed $S U(1,1)$ algebra under the Poisson bracket operation and $I(t)$ was chosen in [10] as the linear combination of these generators. Generalizing the LR-invariant from the Lie-algebraic point of view, we expect that if any time-dependent Hamiltonian be the combination of the generators of any closed algebra, an invariant would be a linear combination of the generators of that closed algebra with time-dependent coefficients.

The integrability of the one-dimensional TDHO is not surprising, because this is a linear system. We know, one-dimensional time-dependent Hamiltonians usually lead to nonintegrability, e.g., a simple pendulum whose length varies in time. Except for the adiabatic or small oscillation approximations, this was the problem posed by Lorentz at the above mentioned Solvay congress and the solutions have the possibility of displaying chaos.

Now a natural question is if there exist one-dimensional time-dependent nonlinear Hamiltonians which are also integrable? In fact, the singular oscillator with a centrifugal force 
potential provides an important example whose kinematics is still within the $S U(1,1)$ algebra. In the second section, we have discussed such a nonlinear Hamiltonian and we have also derived its invariant from our new Lie-algebraic interpretation of the LR-invariant. Using this Hamiltonian we have constructed an integrable, area preserving, nonlinear map and derived its exact invariant with a knowledge of its fixed points.

We have determined the classical time-evolution (Perron-Frobenius) operator following a recent method elucidated by Rau [1]. This method was originally used for solving the quantum Liouville-Bloch equation, but we have applied this in the classical case and have been able to show the one-to-one correspondence between this nonlinear map and the linear map (derived from the Hamiltonian of the one-dimensional TDHO). In the third section, we have studied the quantum dynamics of the nonlinear map and again we have used Rau's method to determine the quantum time-evolution operator. This identical approach to classical and quantum dynamics helps us to show, rather trivially, the exact one-to-one correspondence between the classical and the quantal. Our study also has the dimension of interpreting the mathematics of the $S U(1,1)$ algebra from a dynamical systems viewpoint. For instance, we derive powers of non-commuting products of exponentials of the $S U(1,1)$ algebra. This reveals when the powers can degenerate to the identity, something that is immediately clear from the dynamics of the underlying system due to the presence of degenerate periodic orbits.

\section{THE CLASSICAL KICKED SINGULAR OSCILLATOR}

First, we will describe the general Hamiltonian of which an important special case constitutes the rest of the paper. For the existence of an invariant for the one-dimensional TDHO, the subsidiary condition Eq. (5) has to be integrated with some initial conditions, that is we should be able to determine a particular solution. The subsidiary condition, given in Eq. (5), is a nonlinear, time-dependent, equation and its integrability is not immediately obvious. If we assume $\rho$ as a position variable, say $q$, then Eq. (5) is the equation of motion corresponding to the Hamiltonian given by,

$$
\mathcal{H}(t)=\frac{1}{2}\left(p^{2}+\frac{k}{q^{2}}\right)+\frac{1}{2} \omega^{2}(t) q^{2}
$$

with $k=1$. This is a time-dependent Hamiltonian, and has also been studied for long: it was studied in part by Lewis and Leach [22, Camiz et. al. and Pedrosa et. al. 23] studied this Hamiltonian quantum mechanically. The new nonlinear force in the system is a 'Centrifugal force' and it appears in many integrable systems, including the celebrated Calogero-Sutherland-Moser [24 26] many-body Hamiltonian.

The Hamiltonian in Eq. (6), is formed by the dynamical variables $\frac{1}{2}\left(p^{2}+\frac{k}{q^{2}}\right)$ and $\frac{1}{2} q^{2}$. These two variables together with $p q$, also form the $S U(1,1)$ closed algebra. Following our algebraic interpretation of $I(t)$, we can assume the invariant of this nonlinear Hamiltonian to be of the form,

$$
I(t)=\frac{1}{2} \alpha(t)\left(p^{2}+\frac{k}{q^{2}}\right)+\frac{1}{2} \beta(t) q^{2}+2 \gamma(t) p q
$$


Again, by the same substitutions and identical procedures we get

$$
I(t)=\frac{1}{2} \rho^{2}\left(p^{2}+\frac{k}{q^{2}}\right)+\frac{1}{2}\left(\dot{\rho}^{2}+\frac{1}{\rho^{2}}\right) q^{2}-\rho \dot{\rho} p q,
$$

with the same subsidiary condition as given in Eq. (5). It is also possible to determine this invariant using time-dependent canonical transformations [27], however, our algebraic method accomplishes this more elegantly. In this case, the equation of motion and the subsidiary condition are the same nonlinear equation, but the fact that we need only one particular solution of this equation to determine the invariant shows the power of the methodology and the integrability of the above Hamiltonian in Eq. (6). In Fig. 1 we show a special case corresponding to $\omega^{2}(t)=1+\cos (\sqrt{2} t)$ where the existence of the invariant is reflected in the regular structures.

\section{A. The integrable discrete system}

Now we use the above to construct a nonlinear integrable map. Integrable discrete systems, or maps, have been intensely studied for some time now and many interesting methods and results have been found. Integrable maps are important for the following reasons. Firstly, studying the dynamics of a mapping is more simpler than the dynamics of a continuous system since it involves direct iteration. Secondly, if one wants to study numerically any integrable continuous system, it is absurd to use non-integrable numerical schemes which destroy the basic properties of the system. Therefore, for numerical studies of any integrable system, one has to find a discrete integrable version of the system. The discrete map we discuss below, or extensions thereof, for instance, may be used in the numerical studies of the Calogero-Sutherland-Moser model. Lastly, we can argue that the discrete systems are more fundamental ones since they contain continuous ones as special limits.

Studying the stroboscopic map of any time-periodic system is always possible, at least numerically. To get an analytical expression a much studied form of the time dependence is: $\omega^{2}(t)=\omega^{2} T \sum_{n} \delta(t-n T)$. The standard map and its quantization as well as an experimental realization of the time-dependence through a periodically pulsed laser field [28] provides a well known example of this kind. The map corresponding to the TDHO is a linear map, which is very simple classically.

Here we will study the map corresponding to the nonlinear system. The Hamiltonian of interest is,

$$
\mathcal{H}(t)=\frac{1}{2}\left(p^{2}+\frac{k}{q^{2}}\right)+\frac{1}{2} \omega^{2} T q^{2} \sum_{n} \delta(t-n T),
$$

and the corresponding Hamilton's equations of motion are,

$$
\begin{aligned}
& \dot{p}=\frac{k}{q^{3}}-\omega^{2} T q \sum_{n} \delta(t-n T), \\
& \dot{q}=p .
\end{aligned}
$$


The equation of motion of the system is given by,

$$
\ddot{q}+\omega^{2} T q \sum_{n} \delta(t-n T)-\frac{k}{q^{3}}=0 .
$$

This equation is the same as that of the subsidiary condition given in Eq. (5), except for the constant $k$. Integrating Hamilton's equations of motion from just after the $n$-th kick to just after the $(n+1)$-th kick and defining new scaled variables

$$
p \rightarrow k^{\frac{1}{4}} T^{-\frac{1}{2}} p, \text { and } q \rightarrow k^{\frac{1}{4}} T^{\frac{1}{2}} q,
$$

the phase space map of the system is,

$$
\begin{aligned}
& q_{n+1}=\sqrt{p_{n}^{2}+\frac{1}{q_{n}^{2}}+q_{n}^{2}+2 p_{n} q_{n},} \\
& p_{n+1}=\frac{p_{n}^{2}+\frac{1}{q_{n}^{2}}+p_{n} q_{n}}{q_{n+1}}-\Omega^{2} q_{n+1},
\end{aligned}
$$

where $\Omega=\omega T$.

Note that the scaling has removed the $k$ dependence, but that the scaling is singular at $k=0$ and in fact this limit leads to the removal of the $1 / q^{2}$ term, as is discussed further below. If we plot this phase space map numerically, it clearly shows its regular behaviour, as illustrated in Fig. 2. This rather complicated looking discrete map has a very simple behaviour, reflecting the fact that both this and a linear map, derivable from a TDHO, have common algebraic antecedents. This linear map is derived from the Hamiltonian in Eq. (9) by simply discarding the $1 / q^{2}$ term (the $k=0$ limit) and is given by

$$
\begin{aligned}
& q_{n+1}=p_{n}+q_{n}, \\
& p_{n+1}=p_{n}-\Omega^{2} q_{n+1} .
\end{aligned}
$$

In fact the property of the linear map that exclusively quasi-periodic or entirely periodic behaviour exists for different values of the parameter is also observed for the nonlinear map. For $0<\Omega^{2}<4$ the motion is bounded and stable and for all other values the motion is unbounded and unstable, in both the maps. The invariant in the case of the linear map describes either an ellipse or a hyperbola, now we determine the invariant of the nonlinear map using the method of LR.

Using Eq. (8) the invariant of this map would be of the form,

$$
I=\rho^{2}\left({p_{n}}^{2}+\frac{1}{q_{n}^{2}}\right)+\left(\dot{\rho}^{2}+\frac{1}{\rho^{2}}\right) q_{n}{ }^{2}-2 \rho \dot{\rho} p_{n} q_{n}
$$

where $\rho$ satisfies the same subsidiary condition as given in Eq. (5), but now $\omega^{2}(t)=$ $\omega^{2} T \sum_{n} \delta(t-n T)$. If we are able to determine any particular solution of the subsidiary condition, then we can use that solution to get the invariant of the map Eq. (12). As we have already mentioned, the equation of motion of this nonlinear kicked system is the same as that of the subsidiary condition for $\rho$, as shown in Eq. (11), therefore if we are able to get any solution of the nonlinear map Eq. (12), that solution should also be the solution 
for $\rho$. The most simple solution corresponds to the fixed-point. Therefore the problem of determining the invariant has been reduced to determining the fixed-point of the nonlinear mapping. The fixed point is at,

$$
\begin{aligned}
& q^{*}=-\frac{2}{\Omega^{1 / 2}\left[4\left(4-\Omega^{2}\right)\right]^{1 / 4}}, \\
& p^{*}=\frac{\Omega^{3 / 2}}{\left[4\left(4-\Omega^{2}\right)\right]^{1 / 4}} .
\end{aligned}
$$

This is a particular solution of $\rho$ and $\dot{\rho}$. Note that unlike the linear map whose fixed point at the origin is independent of the system parameters, the fixed points here move with the parameter and as the system approaches instability $\left(\Omega^{2} \rightarrow 4\right)$, they approach infinity. Using these solutions, we get the invariant of the mapping within an arbitrary multiplicative constant as,

$$
I=p_{n}^{2}+\frac{1}{q_{n}^{2}}+\Omega^{2}\left(q_{n}^{2}+q_{n} p_{n}\right) .
$$

The lack of a time subscript on $I$ indicates its constancy. This invariant is valid even when the motion is classically unstable.

\section{B. The classical evolution operator}

We would like to study the nonlinear map from a point of view that simultaneously explains its dynamics as well as sets the stage for quantum mechanical work. Here we introduce the classical time-evolution operator for studying the dynamics of the system. For a given dynamical variable, say $V$, the corresponding Liouville operator is denoted by $L_{V}$ and it is defined as $L_{V} \equiv\{V, *\}$, where $\{*, *\}$ denotes the usual Poisson bracket.

We know the dynamical equation for any dynamical variable, say $f$, is given by,

$$
\frac{d f}{d t}=-\{H, f\} \equiv-L_{H} f
$$

where $H$ is the Hamiltonian of the system and $L_{H}$ is the Liouville operator corresponding to $H$. Let us define $A \equiv \frac{1}{2}\left(p^{2}+\frac{k}{q^{2}}\right), B \equiv \frac{1}{2} q^{2}$ and $\{A, B\}=-p q \equiv-2 C$, i.e., $C=-\frac{1}{2} p q$. The triad $(A, B, C)$ form the closed $S U(1,1)$ algebra. Then we can write,

$$
L_{H}=\left[L_{A}+\omega^{2} T L_{B} \sum_{n} \delta(t-n T)\right],
$$

where $L_{A}$ and $L_{B}$ are the Liouville operators corresponding to $A$ and $B$ respectively. These Liouville operators together with $L_{C}$, the Liouville operator corresponding to $C$, forms the same $S U(1,1)$ algebra as that of $(A, B, C)$ but under the Lie bracket operation, i.e., $\left[L_{A}, L_{B}\right]=-2 L_{C},\left[L_{C}, L_{A}\right]=L_{A}$ and $\left[L_{C}, L_{B}\right]=-L_{B}$.

From Eq. (17), the dynamical equation for the classical time-evolution of any arbitrary function $f$ on the phase space would be,

$$
\frac{d f}{d t}=-L_{H} f=-\left[L_{A}+\omega^{2} T L_{B} \sum_{n} \delta(t-n T)\right] f .
$$


Integrating the above equation Eq. (19) in between the time $t=0$ to $t=T$, we get the classical time-evolution operator from just after zero time to just after the first kick. We write $f(q(t=T), p(t=T))=F f(q(t=0), p(t=0))$ where

$$
F=\exp \left(-\omega^{2} T L_{B}\right) \exp \left(-L_{A} T\right) .
$$

This Perron-Frobenius operator can be regarded as the classical Flouquet operator. To understand the dynamics for time $n$, we have to determine the power $F^{n}$. The one parameter Abelian group of the powers completely specifies the dynamics at all time. However, $F$ is itself a product of the exponential of two non-commuting operators which do not even commute with their commutator $L_{C}$. We can write $F$ in a single exponential form, following an earlier work of Truax [29], but that form is so involved that it becomes difficult to extract the essentially simple dynamics of the system.

We apply a recent operator method, referred to earlier [1], to derive the classical timeevolution operator at any time. The general procedure of this method is simple to describe. If we have any general time-dependent Hamiltonian of the form given by,

$$
\mathcal{H}(t)=\sum_{i=1}^{n} a_{i}(t) H_{i},
$$

where $\left(a_{i}(t), i=1, \ldots, n\right)$ are a set of linearly independent general complex function of time and the dynamical variables $\left(H_{i}, i=1, \ldots, n\right)$ are the generators of any $n$-dimensional closed Lie algebra. Corresponding Liouville operators $\left(L_{H_{i}}, i=1, \ldots, n\right)$ would also form that same algebra. Then the classical time-evolution operator $F(t)$ can be expressed in the product form :

$$
F(t)=\prod_{j=1}^{n} \exp \left[b_{j}(t) L_{H_{j}}\right]
$$

Therefore, in the classical case we can start with the time-evolution operator of the form :

$$
F(t)=\exp \left[X(t) L_{B}\right] \exp \left[Y(t) L_{C}\right] \exp \left[Z(t) L_{A}\right]
$$

where $X(t), Y(t)$ and $Z(t)$ are real functions of time. From the initial condition $F(0)=\mathbf{1}$, we have $X(0)=Y(0)=Z(0)=0$. Now, substituting this product form of $F(t)$ in Eq. (19), and repeatedly applying the Campbell-Baker-Hausdorff $(\mathrm{CBH})$ formula we can cast it into a form such that $F(t)$ is pushed to the extreme right in the LHS of the equation Eq. (19). This yields a set of first order differential equations for the introduced functions of time :

$$
\begin{aligned}
\dot{X} & =-X^{2}-\omega^{2} T \sum_{n} \delta(t-n T), \\
\dot{Y} & =2 X \\
\dot{Z} & =-e^{-Y} .
\end{aligned}
$$

In the equation Eq. (23) we can choose the exponential operators in different orders, but we found that this leads to sets of differential equations whose solutions may not even exist for such kicked systems. 
We now treat the case of delta kicks. Integrating the above equations in between two consecutive kicks and defining

$$
x=T X, y=Y, \text { and } z=\frac{Z}{T},
$$

we get a nonlinear mapping, a "coefficient" mapping for the new dimensionless variables $(x, y, z)$ :

$$
\begin{aligned}
& x_{n+1}=\frac{x_{n}}{1+x_{n}}-\Omega^{2}, \\
& y_{n+1}=y_{n}-\log \left[\left(1+x_{n}\right)^{2}\right], \\
& z_{n+1}=z_{n}+\frac{1}{\Omega^{2}}\left(x_{n+1}-x_{n}\right) .
\end{aligned}
$$

To be explicit the $n$-th power of the operator $F$ is

$$
F^{n}=\exp \left[x_{n} T L_{B}\right] \exp \left[y_{n} L_{C}\right] \exp \left[z_{n} L_{A} / T\right] .
$$

From the initial condition $F^{0}=\mathbf{1}$, we have $x_{0}=y_{0}=z_{0}=0$. The time development is now entirely buried in the scalar functions $x_{n}, y_{n}$, and $z_{n}$.

The most important of the recursion equations in Eq. (25) is the first one. We note that this equation viewed as a transformation is a special case of the "bilinear" conformal transformation in complex analysis or a special case of the projective group $P S L(2, \mathbb{R})$. We solved this nonlinear map by constructing an auxiliary two dimensional linear map. This is not entirely surprising as lurking behind the one dimensional nonlinear singular oscillator is a two dimensional linear one. This gives a new insight into the often stated close relationship between the harmonic oscillator and the singular oscillator.

Before we solve these equations explicitly we point out the following interesting fact. Consider the Hamiltonian

$$
H(q, p, t)=q p^{2}+\Omega^{2} q \sum_{n} \delta(t-n)
$$

written in terms of dimensionless canonical variables. The triad $\left(q p^{2}, q, q p\right)$ form the algebra $S O(2,1)$ under the Poisson bracket operation and this algebra is locally isomorphic to $S U(1,1)$ 30,31. Then the resulting map for $q_{n}$ and $p_{n}$ is

$$
\begin{aligned}
& p_{n+1}=\frac{p_{n}}{1+p_{n}}-\Omega^{2}, \\
& q_{n+1}=q_{n}\left(1+p_{n}\right)^{2} .
\end{aligned}
$$

Thus if we identify $x_{n} \equiv p_{n}$ and $y_{n} \equiv-\log \left(q_{n}\right)$ we get the first two of the recursion relations in Eq. (25). We note that the $S O(2,1)$ algebra underlies the Morse oscillator [32]. Therefore the coefficient map also has an algebraic basis and in fact one that is practically identical to the original one.

We return now to solve Eq. (25). First we define $s_{n} \equiv 1+x_{n}$, and in terms of $s_{n}$, Eq. (25a) becomes

$$
s_{n+1}=\eta-\frac{1}{s_{n}}
$$


where $\eta \equiv 2-\Omega^{2}$, and from the initial condition $x_{0}=0$, we have $s_{0}=1$. Construct the auxiliary linear map:

$$
\left(\begin{array}{l}
a_{n+1} \\
b_{n+1}
\end{array}\right)=\left(\begin{array}{cc}
\eta & -1 \\
1 & 0
\end{array}\right)\left(\begin{array}{l}
a_{n} \\
b_{n}
\end{array}\right)
$$

with initial conditions $a_{0}=b_{0}=1$. We identify $s_{n} \equiv a_{n} / b_{n}$.

To get the general form of $s_{n}$, we have to diagonalize the matrix $M \equiv((\eta,-1),(1,0))$. The eigenvalues of $M$ are,

$$
\lambda_{ \pm}=\frac{1}{2}\left(\eta \pm \sqrt{\eta^{2}-4}\right) .
$$

Whether $\lambda_{ \pm}$is real or complex is dependent on $\eta$. Therefore just as for the linear map we have to study separately three different regions for the parameter $\eta$, these are :

Case 1: $\eta^{2}<4$, i.e., $0<\Omega^{2}<4$.

In this case the eigenvalues of $M$ are complex-conjugate of each other, i.e., we can write

$$
\lambda_{ \pm}=\exp ( \pm i \sigma), \quad \text { where } \sigma \equiv \cos ^{-1}(\eta / 2)=\cos ^{-1}\left(\frac{2-\Omega^{2}}{2}\right)
$$

The dynamics is stable and bounded. After diagonalizing the matrix $M$, we determine $a_{n}$ and $b_{n}$ to finally get:

$$
x_{n}=\cos \sigma-\sin \sigma \tan \left[(2 n-1) \frac{\sigma}{2}\right]-1 .
$$

In terms of $x$ we can obtain the solution for $y$ and $z$ as :

$$
\begin{aligned}
& y_{n}=-2 \sum_{k=0}^{n-1} \log \left|1+x_{k}\right|, \\
& z_{n}=\frac{x_{n}}{\Omega^{2}}
\end{aligned}
$$

When $\sigma=2 \pi m / N$, where $m$ and $N$ are coprime integers, one sees, after some algebra, that

$$
x_{n}=x_{n+N}, \quad y_{n}=y_{n+N}, \quad z_{n}=z_{n+N} .
$$

Therefore for these particular values of $\sigma$ (or of the corresponding value of $\Omega$ ), the above three-dimensional map for the coefficients is exactly periodic. To be explicit at these values of the parameter

$$
F^{N}=\mathbf{1}
$$

the Perron-Frobenius operator becomes unity at time $N$. The quantum equivalence to be discussed below will be complete and the Flouquet unitary operator will become unity at the same time. Fig. 3 shows the coefficients for a periodic case. Since the coefficient $z$ is proportional to $x$, it would follow the same behaviour as $x$. This map in Eq. (12) would also 
be periodic with the same period, and corresponds to the phase space shown in Fig. 2(a). For other values of $\Omega$, this map is quasi-periodic. Equivalently, the phase space map Eq. (12) also displays the above behaviour for corresponding values of $\Omega$. This shows that our operator approach for studying the classical map is in one-to-one correspondence with the

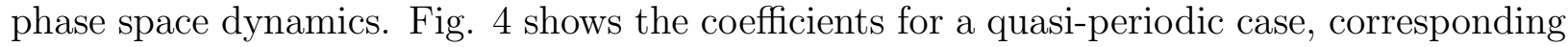
to the phase space in Fig. 2(b). Again the behaviour of $z$ is same as $x$.

Case 2: $\eta^{2}>4$, i.e., $\Omega^{2}>4$ or $\Omega^{2}<0$.

We can divide this case into two parts. They are :

(a) $\eta>2$, i.e., $\Omega^{2}<0$ :

In this part the eigenvalues of $M$ are real and positive. Therefore we can take

$$
\lambda_{ \pm}=\exp ( \pm \sigma), \text { where } \sigma \equiv \cosh ^{-1}(\eta / 2)=\cosh ^{-1}\left(\frac{2-\Omega^{2}}{2}\right) .
$$

In this part the dynamics is unstable and unbounded (hyperbolic). Again following procedures as outlined above, we get

$$
x_{n}=\cosh \sigma+\sinh \sigma \tanh \left[(2 n-1) \frac{\sigma}{2}\right]-1 .
$$

In this case the solution for $y$ and $z$ would also be the same as that given in Eq. (33). However, the basic properties of $x, y$ and $z$ would change due to the unstable and unbounded dynamics, and this is evident from the above equations. For large $n$, both $x$ and $z$ asymptotically reach a constant value that depends on the magnitude of $\Omega$.

$$
x_{\infty}=\lambda_{+}-1, \quad z_{\infty}=x_{\infty} / \Omega^{2} .
$$

However, $y$ would increase linearly with $n$, when $n$ is large.

(b) $\eta<-2$, i.e., $\Omega^{2}>4$.

Here both the eigenvalues of $M$ are real and negative. Thus we can take

$$
\lambda_{ \pm}=-\exp ( \pm \sigma) \text { where } \sigma \equiv \cosh ^{-1}(|\eta| / 2)=\cosh ^{-1}\left(\frac{\left|2-\Omega^{2}\right|}{2}\right) .
$$

In this part the dynamics is still unstable and unbounded; it corresponds, in the linear map, to hyperbolic fixed point with reflections. On following the above procedures, we get

$$
x_{n}=-\cosh \sigma-\sinh \sigma \operatorname{coth}\left[(2 n-1) \frac{\sigma}{2}\right]-1 .
$$

The solutions for $y$ and $z$ still remain the same, and their behaviour for large values of $n$ are qualitatively same as that of the previous part.

Case 3: $\eta^{2}=4$, i.e., $\Omega^{2}=0$ or $\Omega^{2}=4$.

These cases correspond to the marginal ones separating the stable and unstable motions. We can divide this case also into two parts. They are : 
(a) $\eta=2$, i.e., $\Omega^{2}=0$.

Here the eigenvalues of $M$ are equal, $\lambda_{ \pm}=1$. The kick is not operating on the system. This implies that in the expression of the time-evolution operator $F, x=y=0$. Therefore $F$ contains only one exponential and hence the coefficient $z$ would increase linearly with time. This can also be seen as a limiting case; as from Eq. (25a), $\left(x_{n+1}-x_{n}\right) \rightarrow-\Omega^{2}$ and the Eq. (25) gives $z_{n}=-n$.

(b) $\eta=-2$, i.e., $\Omega^{2}=4$.

Again, the eigenvalues of $M$ are equal, but now $\lambda_{ \pm}=-1$. We can get the solution for the coefficients quite easily and these are given by,

$$
\begin{aligned}
& x_{n}=-\frac{2 n+1}{2 n-1}-1, \\
& y_{n}=-2 \log |2 n-1|, \\
& z_{n}=\frac{x_{n}}{\Omega^{2}} .
\end{aligned}
$$

For large $n$, the coefficients $x$ and $z$ asymptotically reach constant values $(-2$ and $-1 / 2)$, while the magnitude of $y$ increases logarithmically. Thus this marginal case straddling the stable and the reflective hyperbolic cases would have power law behaviors in time for phase space variables.

\section{QUANTUM DYNAMICS OF THE KICKED SINGULAR OSCILLATOR}

Quantum mechanical studies of the time-dependent singular oscillator have been carried out for some time now, for instance in [23] where complete analytical solutions were given. We exploit our algebraic method to the special case of the kicked oscillator to lay bare properties such as exact periodicity and quasi-periodicity. In fact, except for a change of terminology, the mathematics is already complete in the previous section.

During our study of the classical nonlinear map, we had introduced the classical timeevolution operator to show the one-to-one correspondence between the nonlinear and the linear map. While classically this is not the usual approach to dynamics, in the case of quantum dynamics, the most natural and popular way is to study the quantum time-evolution operator $\hat{U}(t)$. Thus our classical approach generalizes most easily to the quantum. Previous work [33] that points out exact quantum-classical correspondence in the case of the $S U(1,1)$ algebra for the coefficients of the invariant is easily understood in our approach.

We define the operators as in the classical case:

$$
\hat{A} \equiv \frac{1}{2}\left(\hat{p}^{2}+\frac{1}{\hat{q}^{2}}\right), \quad \hat{B} \equiv \frac{1}{2} \hat{q}^{2}, \quad \text { and }[\hat{A}, \hat{B}]=-\frac{i}{2} \hbar(\hat{p} \hat{q}+\hat{q} \hat{p}) \equiv 2 \hbar \hat{C},
$$

where $(\hat{A}, \hat{B}, \hat{C})$ form the closed $S U(1,1)$ algebra which is given by $[\hat{A}, \hat{B}]=2 \hbar \hat{C},[\hat{C}, \hat{A}]=$ $\hbar \hat{A}$ and $[\hat{C}, \hat{B}]=-\hbar \hat{B}$. Among these operators $\hat{C}$ is anti-hermitian and the other two are hermitian. In terms of these operators our Hamiltonian would be,

$$
\hat{\mathcal{H}}(t)=\hat{A}+\omega^{2} T \hat{B} \sum_{n} \delta(t-n T),
$$


The time-evolution operator $\hat{U}(t)$ satisfies the equation,

$$
i \hbar \frac{d \hat{U}(t)}{d t}=\hat{\mathcal{H}}(t) \hat{U}(t)
$$

where $\hat{U}(0)=\mathbf{1}$ and $\hat{U}(t)$ is unitary. The quantum Flouquet operator $\hat{U}(t=T)$, which we denote simply as $\hat{U}$, is given by

$$
\hat{U}=\exp \left(-\frac{i}{\hbar} \omega^{2} T \hat{B}\right) \exp \left(-\frac{i}{\hbar} T \hat{A}\right) .
$$

For the complete dynamics, as usual, we have to determine the powers $\hat{U}^{n}$. Again we apply Rau's [1] method for the derivation of the time-evolution operator at any arbitrary time. We start with the time-evolution operator of the form,

$$
\hat{U}(t)=\exp \left[\frac{i}{\hbar} X(t) \hat{B}\right] \quad \exp \left[\frac{1}{\hbar} Y(t) \hat{C}\right] \quad \exp \left[\frac{i}{\hbar} Z(t) \hat{A}\right]
$$

where $X(t), Y(t)$ and $Z(t)$ are real functions of time, so that $\hat{U}(t)$ remains unitary. From the initial condition $\hat{U}(0)=1$, we have $X(0)=Y(0)=Z(0)=0$. Substituting the above $\hat{U}(t)$ in Eq. (40), following identical procedures as given for the classical dynamics, we get

$$
\begin{aligned}
\dot{X} & =-X^{2}-\omega^{2} T \sum_{n} \delta(t-n T), \\
\dot{Y} & =2 X, \\
\dot{Z} & =-e^{-Y} .
\end{aligned}
$$

These set of equations for the quantum coefficients are identical to the equations for the classical coefficients as given in Eq. (24). Therefore we would get an identical map for the quantum coefficients as for the classical coefficients Eq. (25). Since these two maps are identical, their stability properties are identical, i.e., quantum dynamics exactly follows its classical counterpart. Thus when the classical Perron-Frobenius operator becomes identity as in Eq. (35) the Flouquet operator also becomes identity. The various cases discussed classically, including the marginal and the reflective hyperbolic cases have exact quantum counterparts. Thus as far as time evolution is concerned the quantal problem is already solved. Issues of eigenstates and spectrum can be tackled similarly, as for example done in [34] for the case of the kicked harmonic oscillator; however we do not pursue this further here.

\section{SUMMARY}

We have studied, both classically and quantum mechanically, a time-dependent onedimensional nonlinear integrable system, namely the kicked harmonic oscillator with a singular potential. By applying our Lie-algebraic interpretation of the LR-invariant, we have determined the exact invariant of that nonlinear system. One can apply this method to determine the invariant of any time-dependent Hamiltonian, formed by the generators of any 
closed algebra. We have also found, as far as we know, a new integrable nonlinear mapping. Here we derived that map from the Hamiltonian formed of the generators of the $S U(1,1)$ algebra. We expect that we can determine integrable two-dimensional mappings from the Hamiltonians formed by the generators of other closed algebras.

We constructed the classical time-evolution operator, or the Perron-Frobenius operator for the nonlinear integrable system, taking advantage of a recent method of solving the time-dependent Schrödinger equation. This brings into relief the extreme quantum-classical correspondence in these systems which have algebraic structures underlying them.

We may speculate whether for time-dependent systems with more than one degree of freedom, which are constructed from elements of some Lie algebra, the nonlinear equations (whether difference or differential) that are the analogue of the coefficient mapping in Eq. (25) are integrable. Further, if there is a connection between the integrability of these equations and that of the original system itself. Our study that began as an attempt to study a nonlinear time-dependent integrable system led us to one that is in many respects related to the harmonic oscillator with time-dependent frequency. We may then ask if we can go beyond this limitation to a more "genuine" form of nonlinearity. 


\section{REFERENCES}

[1] A. R. P. Rau, Phys.Rev.Lett., 81, 4785 (1998).

[2] A. de Sonza Dutra, C. F. de Sonza and L. C. Albuquerque, Phys.Lett.A, 156, 371 (1991).

[3] C. F. Lo, Phys.Rev.A, 45, 5262 (1992).

[4] M. V. Berry, Proc.R.Soc.A, 392, 45, (1984).

[5] A. Shapere and F. Wilczek, (Eds.) Geometric phases in physics, Advanced series in mathematical physics, Vol.5 (World Scientific, Singapore, 1989).

[6] S. J. Wang, Phys.Rev.A, 42, 5103; 5107 (1990).

[7] D. J. Moore, Phys.Rep., 210, 1 (1991).

[8] H. R. Lewis Jr., Phys.Rev.Lett., 18, 510 (1967).

[9] H. R. Lewis Jr., J.Math.Phys., 9, 1976 (1968).

[10] H. R. Lewis Jr. and W. B. Riesenfeld, J.Math.Phys., 10, 1458 (1969).

[11] W. Paul, Rev.Mod.Phys., 62, 531 (1990).

[12] L. S. Brown, Phys.Rev.Lett., 66, 527 (1991).

[13] R. Bengtsson and J. D. Garret, in : Collective phenomenon in atomic nuclei, International review of nuclear physics (World Scientific, Singapore, 1984).

[14] S. Ayik and W. Nörenberg, Z.Phys.A, 309, 121 (1982).

[15] V. I. Arnold, Mathematical Methods of Classical Mechanics, 2nd ed. (Springer-Verlag, 1989).

[16] A. J. Lichtenberg and M. A. Lieberman, Regular and Chaotic Dynamics, 2nd ed. (Springer-Verlag, 1992).

[17] A. Einstein, Inst. interm. phys. Solvay, Conseil phys., Rapports. et discussions, 1, 450 (1911).

[18] M. Kruskal, J. Math. Phys., 3, 806 (1962).

[19] W. E. Milne, Phys.Rev., 35, 863 (1930); C. H. Greene, A. R. P. Rau and U. Fano, Phys.Rev.A, 26, 2441 (1982); U. Fano and A. R. P. Rau, Atomic Collisions and Spectra (Academic Press, 1986).

[20] C. J. Eliezer and A. Gray, S. I. A. M. 30, 463 (1976).

[21] N. J. Günther and P. G. L. Leach, J. Math. Phys. 18, 572 (1977).

[22] H. Ralph Lewis and P. G. L. Leach, J.Math.Phys., 23(1), 165 (1982).

[23] P. Camiz, A. Geradi, C. Marchioro, E. Presutti and E. Scacciatelli, J.Math.Phys., 12, 2040 (1971); I. A. Pedrosa, G. P. Serra and I. Guedes, Phys.Rev.A, 56, 4300 (1997).

[24] F. Calogero, J.Math.Phys., 12, 419 (1971).

[25] B. Sutherland, J.Math.Phys., 12, 246 ; 12, 251 (1971).

[26] J. Moser, Adv.Math., 16, 197 (1975).

[27] I. A. Pedrosa, J.Math.Phys.(N. Y), 28, 2662 (1987).

[28] F. L. Moore, J. C. Robinson, C. F. Bharucha, Bala Sundaram and M. G. Raizen, Phys.Rev.Lett., 75, 4598 (1995).

[29] D. Rodney Truax, Phys.Rev.D, 31, 1988 (1985).

[30] A. Perelomov, Generalized Coherent States and Their Applications (Springer-Verlag, 1986).

[31] J. F. Cornwell, Group Theory In Physics, Vol. 2 (Academic Press, 1984).

[32] M. Berrondo and A. Palma, J.Phys.A, 13, 773 (1980).

[33] S. J. Wang, W. Zuo, A. Weiguny and F. L. Li, Phys.Lett.A, 196, 7 (1994). 
[34] M. V. Berry, N. L. Balazs, A. Voros and M. Tabor, Ann. Phys. (N.Y.), 122, 26 (1979); A. Lakshminarayan and N. L. Balazs, Ann. Phys. (N.Y.), 212, 220 (1991). 


\section{FIGURES}

FIG. 1. Stroboscopic picture of the time-dependent harmonic oscillator with a singular perturbation; the integrable behaviour is evident. All quantities shown are dimensionless

FIG. 2. (a) Period-9 orbit of the nonlinear map for the case $\Omega^{2}=2\left[1-\cos \left(\frac{2 \pi}{9}\right)\right] \approx 0.4679$, (b) quasi-periodic orbit for the case $\Omega^{2}=2.43$. All quantities shown are dimensionless

FIG. 3. The coefficients of the time-evolution operator showing the period-9 behaviour for the

same $\Omega^{2}$ as in Fig. 2(a). Shown are the $x$ (a) and $y(\mathrm{~b})$ coefficients. All quantities shown are dimensionless

FIG. 4. The coefficients of the time-evolution operator showing the quasi-periodic behaviour

for the same $\Omega^{2}$ as in Fig. 2(b). Shown are the $x$ (a) and $y$ (b) coefficients. All quantities shown are dimensionless 


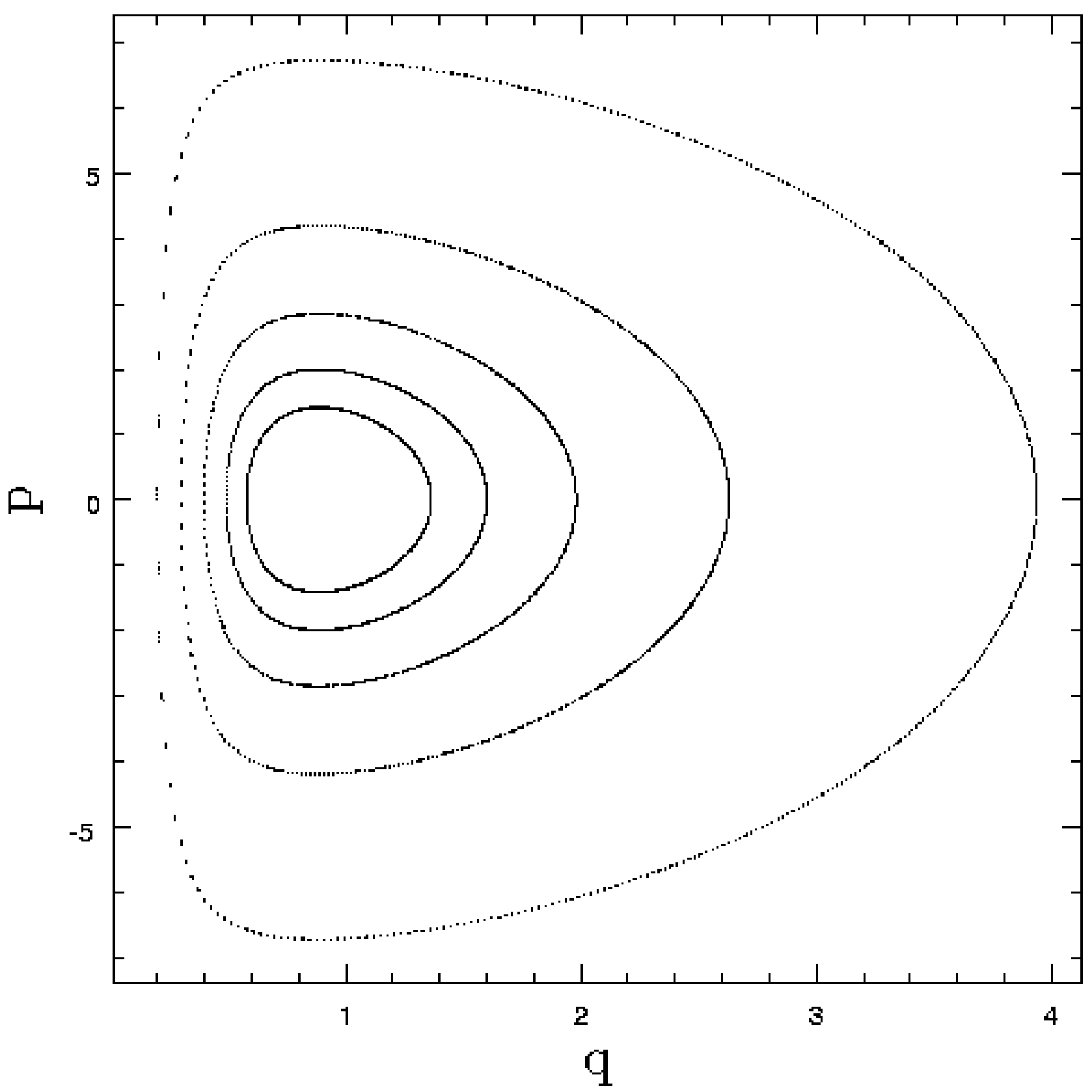



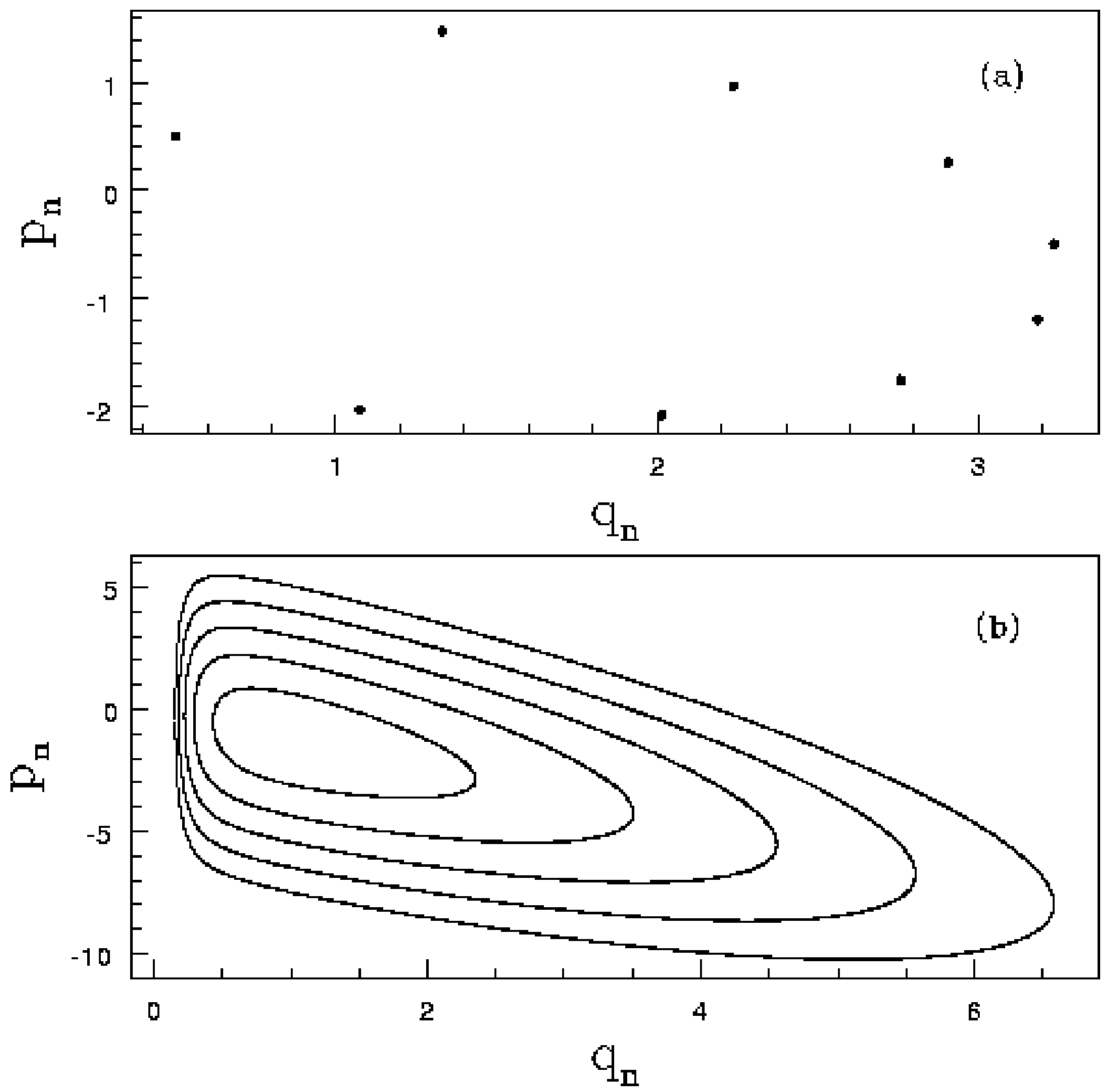


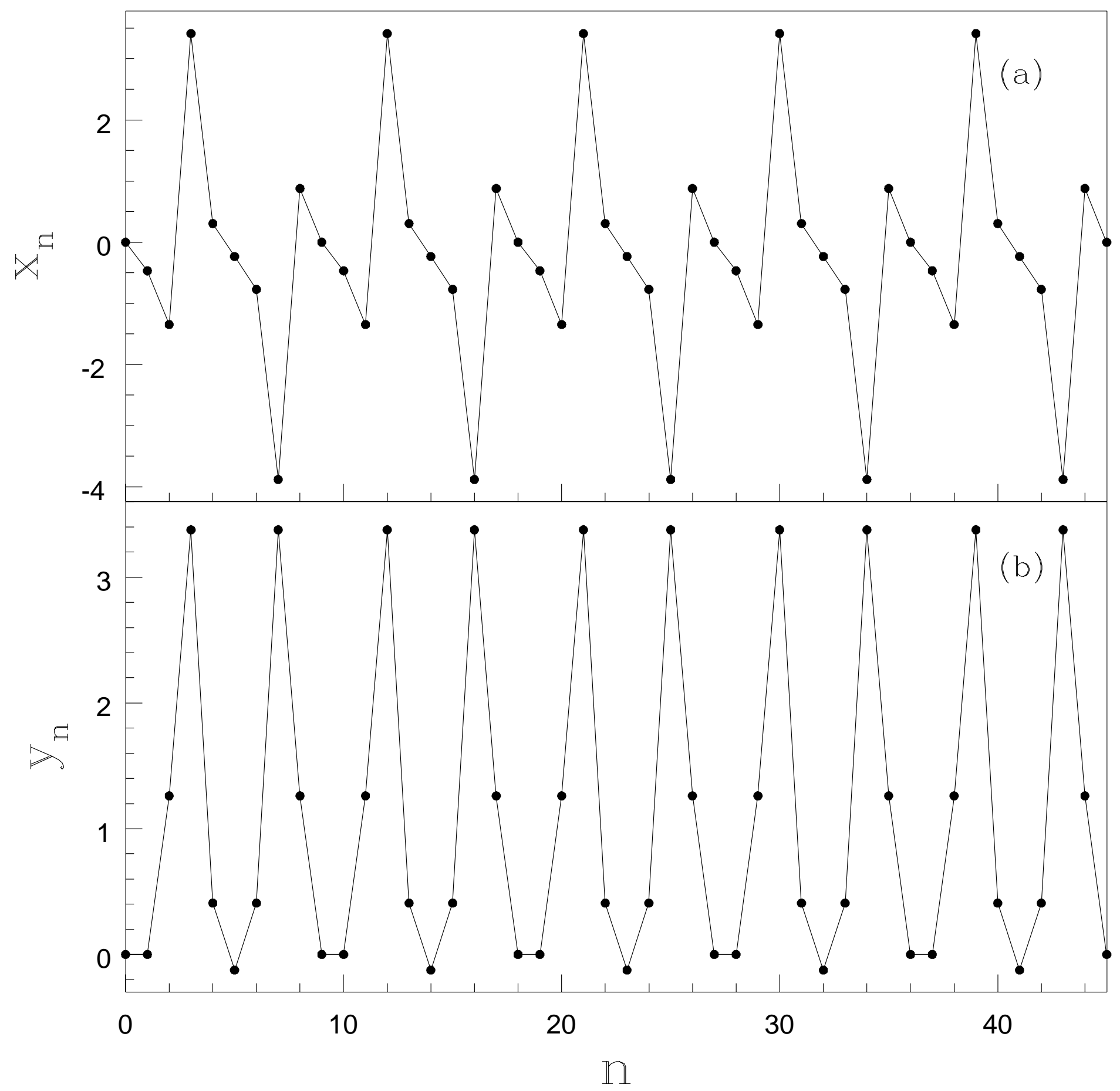




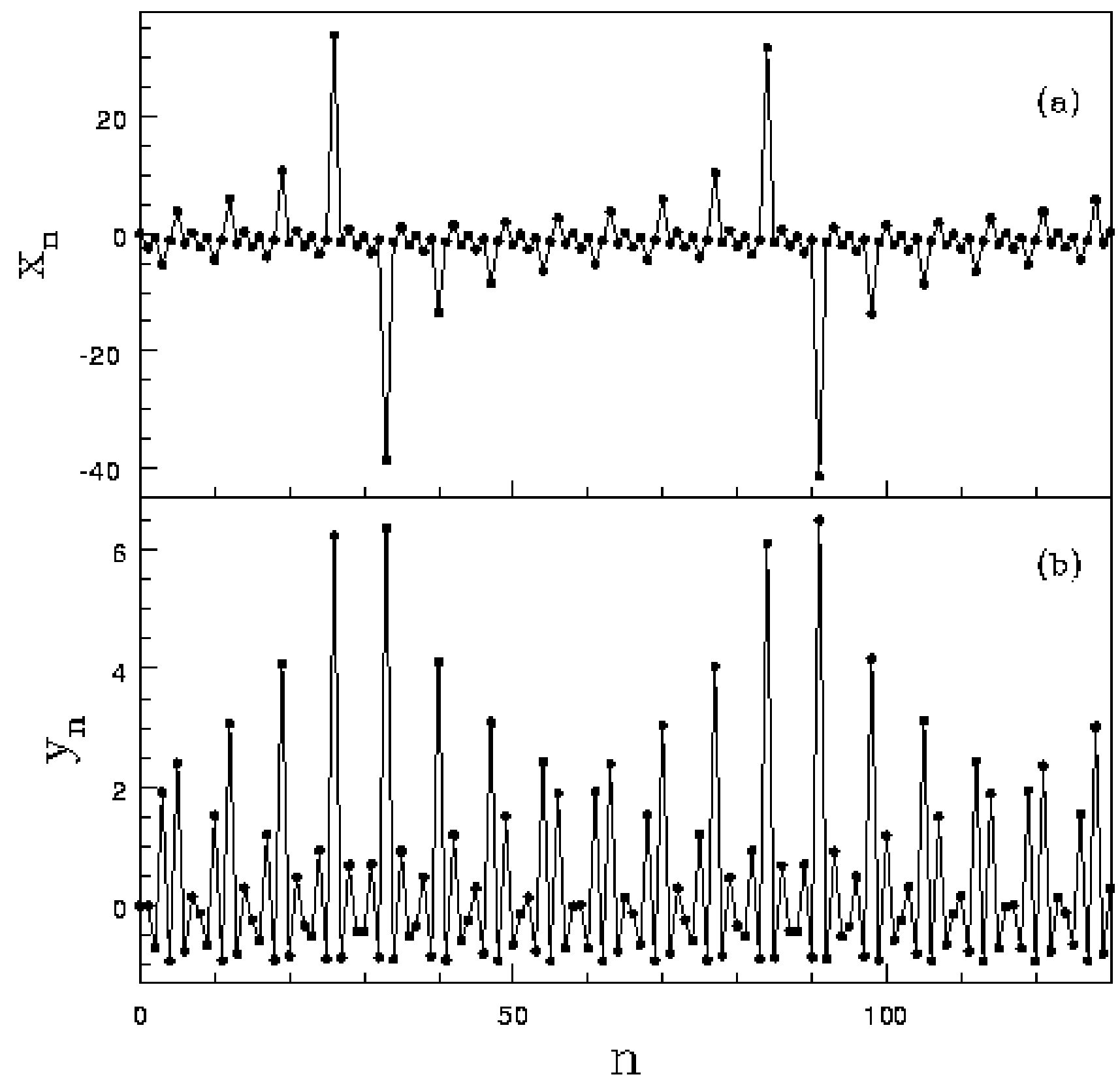

